\title{
The new Brazilian Lightning Detection Network: First Results
}

\author{
Osmar Pinto Jr., Kleber P. Naccarato and Iara R. C. A. Pinto \\ Atmospheric Electricity Group (ELAT) \\ Brazilian Institute of Space Research - INPE \\ E-mail: osmar@dge.inpe.br
}

\begin{abstract}
A new lightning detection network using WeatherBug Total Lightning Sensors (WTLS) is being deployed in Brazil. The network can acquire detailed signals emitted from both IC and CG flashes and continuously sends information to a central server. A WTLS is composed of an antenna, a global positioning system (GPS) receiver, a high-accuracy GPS-based timing circuit, a digital signal processor (DSP), and onboard storage and internet communication equipment. The sensor is a wideband system with detection frequency ranging from $1 \mathrm{~Hz}$ to $12 \mathrm{MHz}$. The wide frequency range enables the sensor to not only detect strong CG strokes, but to also detect weak IC pulses. The sensor records whole waveforms of each flash and sends them back, in compressed data packets, to the central server. Instead of using some waveform parameters, the whole waveforms are used in locating the flashes and differentiating between IC and CG strokes. A 75-sensor network is being installed in the South, Southeast, Center and Northeast regions of the country and should operated simultaneously with the integrated lightning detection network (RINDAT), which is composed by 34 lowfrequency (LF) sensors using Vaisala technology, covering the Southeast and parts of the South and Center regions of the country. Details of the new network configuration and its main applications will be described.
\end{abstract}

Keywords - lightning; lightning networks.

\section{INTRODUCTION}

WeatherBug Total Lightning Sensors (WTLS) are an integrated in-cloud (IC) and cloud-to-ground (CG) lightning detector that uses wide-band technology to capture both IC and CG strokes. It is well known that in-cloud lightning detection at high efficiencies is critical for advanced forecasting of severe weather phenomena such as tornadoes, damaging downburst winds, flash floods, wind shear, and deadly CG lightning strikes that often follow 5 to $30 \mathrm{~min}$ after IC strokes begin. The WTLS provides stroke or flash time, latitude, longitude, peak current for $\mathrm{CG}$, peak radiated field normalized to $30 \mathrm{~km}$ for IC, and IC/CG classification. Additionally, the actual captured waveforms of the events can be accessed. The peak current is given in amperes and the waveforms are given in volts per meter.

The WTLS use a time-of-arrival method of detection. Multiple sensors are used to locate and verify individual strokes adding precision to the system. Time-of-arrival methodology also enables simpler calibration than direction finding. Direction-finding technology is limited by bearing errors while time-of-arrival techniques are limited by timing errors. It is much easier to calibrate timing than bearing, reducing potential error in locating lightning and improving location accuracy. With GPS timing and location present capabilities, the time-of-arrival methodology has become increasingly more accurate than magnetic direction finding.

The WTLS were designed and built within advanced electronics and components all in a compact unit. The units were made trying to reduce system noise and to broaden the frequency range in order to create an integrated unit capable of detecting both ground and cloud strokes with very high detection efficiencies. The sensors operate in the $1 \mathrm{~Hz}$ to 12 $\mathrm{MHz}$ range in order to detect both $\mathrm{CG}$ and IN lightning activity. Waveforms for every stroke are transmitted from the site and centrally archived. The entire waveforms are used to flash discrimination using a cross-correlation methodology. The fact that most cloud pulses are much weaker than return strokes, seeing them requires low noise electronics. More details about these sensors are found in [1] and [2].

\section{NEW BraZiLIAN LightNing Detection NETWORK}

The first lightning detection network in Brazil was installed in 1989 composed by 4 LPTAS sensors. Since that time the network grows considerably to achieve almost 50 sensors in 2005 , then reducing to approximately 35 sensors in 2010 . The network called RINDAT was intended to detect CG flashes. A review about the history of the lightning detection networks in Brazil can be found in [3] and [4].

Since December 2010, INPE starts to deploy a WTLS in Southeastern Brazil that was called preliminarily the Brazilian Total Lighting Network (BTLN). The main goals of the network are: to detect IC flashes with high detection efficiency and to introduce a new lightning detection technology in Brazil in the same way that has been done in USA and Europe, where two or more lightning detection networks operates simultaneously. The idea is to generate distinct lightning data (that come from different sources) in order to improve the lightning research and to allow a wider use of the information for several other institutions and companies.

Figure 1 shows the location of the first 12 sensors BTLN. The installation of these sensors started in December 2010 and was concluded in May 2011. Up to July 2012, more 60 sensors should be installed covering the South, Southeast, Center and Northeast regions of the country. At the time of this conference 
(November 2011), the whole Southeast region would be covered (an update of the network deployment will be given during the conference).

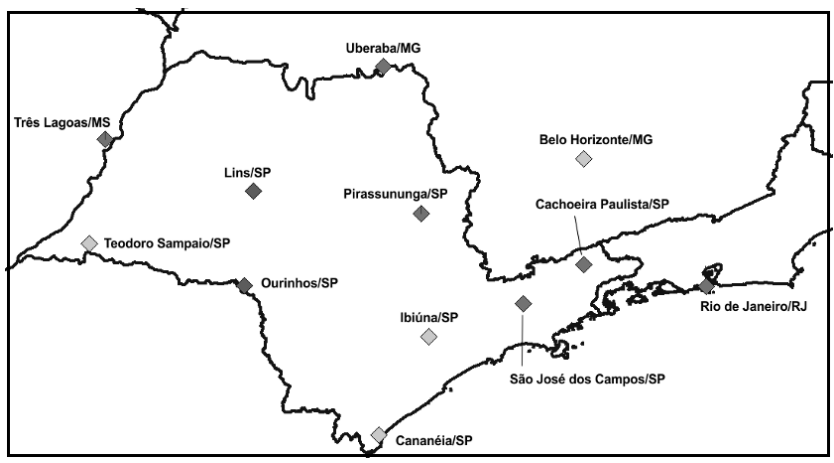

Figure 1. Location of the first 12 sensors of the BTLN. The sensors were operational in May 2011.

For March 2011, the BTLN has detected 300,176 lightning strokes $(\mathrm{CG}+\mathrm{IC})$ over the Southeastern Brazil. From those lightning data, 182,126 are IC discharges $(60,7 \%)$ and 118,050 $(39,3 \%)$ are CG strokes. The IC/CG ratio is in reasonable agreement with a previous study in Brazil [5] over Rondonia and Southeast Brazil based on total lightning data from LIS and CG lightning data from BLDN and RINDAT networks.

Figure 2 shows separately the CG and IC strokes detected by BTLN for 48hr of data: March 28 and 29, 2011. For these 2 days, the total of 69,006 lightning strokes are detected: IC discharges 43,721 or $63,4 \%$ (IC) and 25,285 or $36,6 \%$ (CG). That the IC/CG ratio for this period was similar to the whole month. We are now working on calibrations and adjustments (fine tune) of the sensors that will allow BTLN to provide polarity and intensity of the CG strokes.

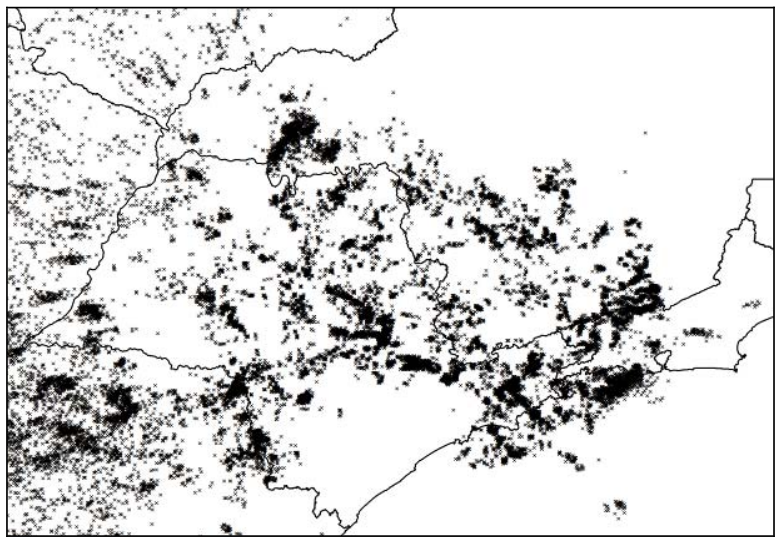

(a)

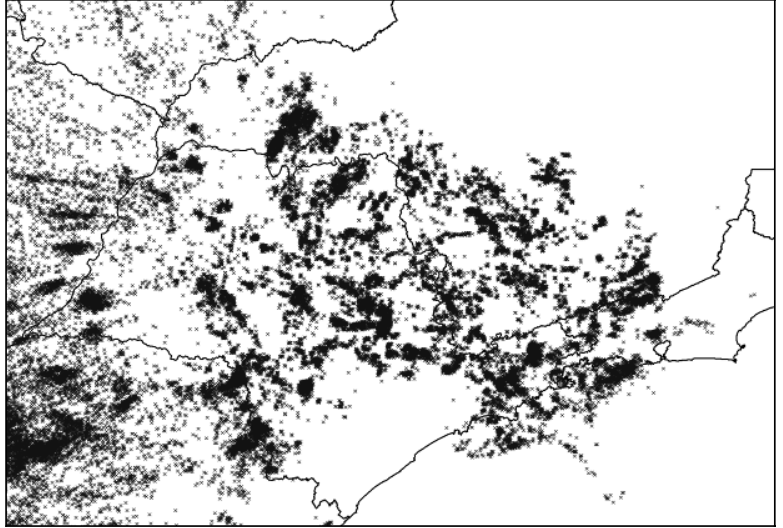

(b)

Figure 2. Sample of total lightning data: (a) CG strokes; (b) IC discharges.

\section{CONCLUSIONS}

This paper presents the first total lightning data provided by the new Brazilian Total Lightning Network (BTLN) in Southeastern Brazil. The results correspond only to one month of data (March 2011) when only 8 sensors are operational. The main conclusion of this work is to show that BTLN is now able to provide IC and CG lightning data for several purposes. The data should be validated in the near future comparing to other independent sources in order to evaluate the detection efficiency and location and discrimination accuracy. At the time of the conference more information will be presented.

\section{ACKNOWLEDGMENT}

The authors thank many institutions that support this new development in Brazil.

\section{REFERENCES}

[1] AWS Convergence Technologies. WeatherBug Total Lightning System: technical overview and network configuration options. White Paper, 2010.

[2] Heckman, S. and Liu, C. The application of total lightning detection and cell tracking For severe weather prediction, Proceedings of the GROUND'2010 and 4rd LPE, CD-ROM, Salvador, 2010.

[3] Pinto Jr., O. The Brazilian lightning detection network: a historical background and future perspectives, Proceedings of SIPDA, Curitiba, 2003.

[4] Pinto Jr., O. Lightning in the tropics, Nova Publishers, 2009.

[5] Pinto Jr., O.; Pinto, I. R. C. A.; Faria, H. H. A comparative analysis of lightning data from lightning networks and LIS sensor in the North and Southeast of Brazil. Geophysical Research Letters, v.30, n.2, 1073, doi:10.1029/2002GL016009, 2003. 